\title{
ARCHIMEDEAN COMPONENTS OF TRIANGULAR NORMS
}

\author{
ERICH PETER KLEMENT, RADKO MESIAR and ENDRE PAP
}

(Received 30 May 2002; revised 10 December 2003)

Communicated by D. Easdown

\begin{abstract}
The Archimedean components of triangular norms (which turn the closed unit interval into an abelian, totally ordered semigroup with neutral element 1) are studied, in particular their extension to triangular norms, and some construction methods for Archimedean components are given. The triangular norms which are uniquely determined by their Archimedean components are characterized. Using ordinal sums and additive generators, new types of left-continuous triangular norms are constructed.
\end{abstract}

2000 Mathematics subject classification: primary 20M05; secondary $03 \mathrm{E} 72$.

Keywords and phrases: semigroup, triangular norm, Archimedean component.

\section{Introduction}

Equipped with a triangular norm as binary operation, the unit interval is an abelian, totally ordered semigroup having 1 as neutral element. Triangular norms were introduced by Schweizer and Sklar [30] in the framework of probabilistic metric spaces (see $[12,31])$, and they were originally used to carry over the triangle inequality from classical metric spaces to this more general setting, following some ideas outlined by Menger [24].

Although no representation of the class of all t-norms is known so far, a well-known fact is that continuous t-norms are just ordinal sums in the sense of Clifford [4] of continuous Archimedean t-norms [23, 31]. However, Archimedean components are not yet fully understood in the case of general (not necessarily continuous) t-norms. The aim of this paper is to contribute to this understanding.

This work was supported by two European actions (CEEPUS network SK-42 and COST action 274) as well as by grants VEGA 1/0273/03 and MNTRS-1866. The authors also thank the referee whose suggestions helped to improve the paper significantly.

(C) 2005 Australian Mathematical Society $1446-7887 / 05 \$ \mathrm{~A} 2.00+0.00$ 
Archimedean components of triangular norms are convex subsemigroups of the unit interval. The relationship between t-norms and their Archimedean components is studied, in particular, the conditions under which a t-norm is uniquely determined by its Archimedean components. This naturally leads to the construction of t-norms from Archimedean components and, subsequently, of Archimedean components themselves.

We characterize the class of triangular norms which are uniquely determined by their Archimedean components (Theorem 4.4). In general, we give explicit formulae for the strongest t-norm having a given system of Archimedean components, and (under some additional assumptions) for the weakest $t$-norm which can be written as an ordinal sum, as well as for the weakest t-norm having among their Archimedean components the given ones. A general construction of $t$-norms from Archimedean components is given, and several construction methods for non-trivial Archimedean components are presented. In particular, the construction suggested in Proposition 5.3 always yields left-continuous Archimedean components generated by continuous, non-increasing additive generators which, in combination with Theorem 2.5 , gives rise to a rich class of left-continuous $t$-norms.

The problems studied here add new insights and construction methods for general t-norms. This contributes to several fields of applications where the need for a deeper understanding of (left-continuous) t-norms has significantly increased over the last years. In many-valued logics based on residuated lattices [11, 13, 18, 34] the conjunction is evaluated by lower semicontinuous functions, which are just leftcontinuous $t$-norms if the set of truth values equals $[0,1]$. Similar is the situation in the field of probabilistic metric spaces, in the general theory of non-additive measures and integrals [21, 29], in chaos theory and dynamical systems [32], and in the modelling of preference structures [8] and of cooperative games [2].

After presenting the most important facts about triangular norms and ordinal sums we introduce and discuss in Section 3 Archimedean classes and components. Section 4 deals with extensions of Archimedean components to triangular norms, paying special attention to uniqueness of extensions. Finally we present some construction methods for Archimedean components.

\section{Triangular norms and ordinal sums}

A triangular norm (t-norm for short) $T$ is a binary operation on the closed unit interval $[0,1]$ such that $([0,1], T)$ is an abelian semigroup with neutral element 1 which is compatible with the natural order $\leq$ on $[0,1]$. Basic examples of $t$-norms are the minimum $T_{\mathbf{M}}$, the product $T_{\mathbf{P}}$, the Łukasiewicz $\mathrm{t}$-norm $T_{\mathbf{L}}$ given by $T_{\mathrm{L}}(x, y)=$ $\max (x+y-1,0)$, and the drastic product $T_{\mathbf{D}}$ with $T_{\mathbf{D}}(1, x)=T_{\mathbf{D}}(x, 1)=x$, and 
$T_{\mathbf{D}}(x, y)=0$ otherwise. Clearly, for each $\mathrm{t}$-norm $T$ we have $T_{\mathbf{D}} \leq T \leq T_{\mathbf{M}}$. For more details on triangular norms we refer to [18].

Several specific notions, properties and constructions from semigroup theory were studied for t-norms, such as the Archimedean property, the cancellation law, nilpotent elements, zero divisors and ordinal sums $[18,31]$. We only recall that a t-norm $T$ is Archimedean if for each $(x, y) \in] 0,1\left[^{2}\right.$ there is an $n \in \mathbb{N}$ with $x_{T}^{(n)}<y$, where $x_{T}^{(n)}$ is defined inductively by $x_{T}^{(1)}=x$ and $x_{T}^{(n+1)}=T\left(x_{T}^{(n)}, x\right)$. We may write $x^{(n)}$ for $x_{T}^{(n)}$ if $T$ is clear from context.

Continuous t-norms turn [0,1] into a topological semigroup [3] (more precisely, into an $I$-semigroup $[7,26,28]$ ). We have the following representations $[18,23,31]$, using the pseudo-inverse $t^{(-1)}:[0, \infty] \rightarrow[0,1]$ of a non-constant non-increasing function $t:[0,1] \rightarrow[0, \infty]$ which is given by

$$
t^{(-1)}(u)=\sup \{x \in[0,1] \mid t(x)>u\}
$$

(using the convention $\sup \emptyset=\inf [0,1]=0$ ). Note that in the case of a continuous, strictly decreasing function $t$ satisfying $t(1)=0$ we have

$$
t^{(-1)}(u)= \begin{cases}v & \text { if } t(v)=u \\ 0 & \text { if } u>t(0)\end{cases}
$$

THEOREM 2.1. A function $T:[0,1]^{2} \rightarrow[0,1]$ is a continuous Archimedean t-norm if and only if there is a continuous, strictly decreasing function $t:[0,1] \rightarrow[0, \infty]$ with $t(1)=0$ such that

$$
T(x, y)=t^{(-1)}(t(x)+t(y)) .
$$

In general, given a (not necessarily continuous) t-norm $T$, each strictly decreasing function $t:[0,1] \rightarrow[0, \infty]$ which is right-continuous in 0 , satisfies $t(1)=0$ and, for all $(x, y) \in[0,1]^{2}, t(x)+t(y) \in \operatorname{Ran}(t) \cup[t(0), \infty]$ such that (1) holds, is called an additive generator of $T$, and it is uniquely determined by $T$ up to a positive multiplicative constant. Thus, Theorem 2.1 states that continuous Archimedean tnorms are characterized by having continuous additive generators. Note that each $\mathrm{t}$-norm possessing an additive generator is necessarily Archimedean.

THEOREM 2.2. A function $T:[0,1]^{2} \rightarrow[0,1]$ is a continuous $t$-norm if and only if there exist a family $\left(T_{\alpha}\right)_{\alpha \in A}$ of continuous Archimedean $t$-norms and a family (]$a_{\alpha}, b_{\alpha}[)_{\alpha \in A}$ of non-empty, pairwise disjoint open subintervals of $[0,1]$ such that $T$ equals the ordinal sum $\left(\left\langle a_{\alpha}, b_{\alpha}, T_{\alpha}\right\rangle\right)_{\alpha \in A}$, that is,

(2) $T(x, y)= \begin{cases}a_{\alpha}+\left(b_{\alpha}-a_{\alpha}\right) T_{\alpha}\left(\frac{x-a_{\alpha}}{b_{\alpha}-a_{\alpha}}, \frac{y-a_{\alpha}}{b_{\alpha}-a_{\alpha}}\right) & \text { if }(x, y) \in\left[a_{\alpha}, b_{\alpha} I^{2} ;\right. \\ \min (x, y) & \text { otherwise. }\end{cases}$ 
Let us now recall some well-known facts about Archimedean t-norms. Each Archimedean t-norm satisfies $T(x, x)<x$ for all $x \in] 0,1[$. The converse is not true, in general. See, for example, [31, page 60] or Example 1 below. However, if a rightcontinuous t-norm $T$ satisfies $T(x, x)<x$ for all $x \in] 0,1[$ then it is Archimedean.

The continuous Archimedean t-norms without zero divisors are called strict, and each of them is isomorphic to the product t-norm $T_{\mathbf{P}}$. Each strict t-norm satisfies the cancellation law on $] 0,1]^{2}$, and each of its additive generators $t$ is unbounded, that is, satisfies $t(0)=\infty$.

The non-strict continuous Archimedean t-norms are called nilpotent, and each of them is isomorphic to the Łukasiewicz t-norm $T_{\mathbf{L}}$. Each nilpotent t-norm $T$ satisfies the cancellation law on its positive domain $\left.\left.T^{-1}(] 0,1\right]\right)$, each of its additive generators $t$ is bounded, that is, satisfies $t(0)<\infty$, and each $x \in] 0,1[$ is both a zero divisor and a nilpotent element of $T$.

As an immediate consequence of these facts, a t-norm $T$ is continuous if and only if it is isomorphic to some ordinal sum of t-norms $\left(\left\langle a_{\alpha}, b_{\alpha}, T_{\alpha}\right\rangle\right)_{\alpha \in A}$ where each summand $T_{\alpha}$ equals either $T_{\mathbf{P}}$ or $T_{\mathbf{L}}$.

Although the structure of continuous t-norms as ordinal sums of continuous Archimedean t-norms has been known for several decades, a deeper study of general (not necessarily continuous) $t$-norms from the semigroup theory point of view only recently gained new momentum. Using the unique infinite dyadic representation of numbers $x=\sum_{n=1}^{\infty} 1 / 2^{x_{n}}$ in $\left.] 0,1\right]$, where $\left(x_{n}\right)_{n \in \mathbb{N}}$ is a strictly increasing sequence of positive integers, the following examples appear in [18]:

EXAMPLE 1. The functions $T_{1}, T_{2}:[0,1]^{2} \rightarrow[0,1]$ defined by

$$
\begin{aligned}
& T_{1}(x, y)= \begin{cases}\sum_{n=1}^{\infty} 1 / 2^{x_{n}+y_{n}} & \text { if }(x, y) \in] 0,1\left[^{2} ;\right. \\
\min (x, y) & \text { otherwise, }\end{cases} \\
& T_{2}(x, y)= \begin{cases}\sum_{n=1}^{\infty} 1 / 2^{x_{n}+y_{n}-n} & \text { if }(x, y) \in] 0,1]^{2} ; \\
0 & \text { otherwise, }\end{cases}
\end{aligned}
$$

are strictly monotone $t$-norms (that is, they satisfy the cancellation law on $] 0,1]^{2}$ ) which are discontinuous at each point $(x, y) \in] 0,1]^{2}$ having at least one coordinate with a finite dyadic representation. We have $T_{1}(x, x)<x$ and $T_{2}(x, x)<x$, respectively, for all $x \in] 0,1$ [, and $T_{2}$ is left-continuous. However $T_{1}$ is Archimedean while $T_{2}$ is not.

Also the exact relationship between general t-norms and ordinal sums of semigroups was clarified recently. Some special semigroups introduced as t-subnorms in [16] and as tosabs in [20] proved to be useful in this context.

DEFINITION 2.3. Let $I$ be a non-empty subinterval of the closed unit interval $[0,1]$. 
(i) A totally ordered abelian semigroup $(I, *)$ where $*$ is bounded from above by the minimum will be called a tosab.

(ii) A tosab $([0,1], *)$ is called a $t$-subnorm.

In [20, Proposition 2.3] it was shown that the only way to obtain a t-norm as an ordinal sum of semigroup operations is to construct certain ordinal sums of tosabs.

PROPOSITION 2.4. Let $([0,1], *)$ be the ordinal sum of a family of semigroups $\left(\left(X_{\alpha}, *_{\alpha}\right)\right)_{\alpha \in A}$, that is, $\left\{X_{\alpha} \mid \alpha \in A\right\}$ is a partition of $[0,1]$ and for all $(x, y) \in[0,1]^{2}$ we have

$$
x * y= \begin{cases}x *_{\alpha} y & \text { if }(x, y) \in X_{\alpha}^{2} \\ \min (x, y) & \text { otherwise. }\end{cases}
$$

Then the operation $*$ is a $t$-norm if and only if each $\left(X_{\alpha}, *_{\alpha}\right)$ is a tosab, if the order on $A$ is compatible with the usual order on $[0,1]$, and if there is an $\alpha_{0} \in A$ such that 1 is the neutral element of $*_{\alpha_{0}}$.

In [16] ordinal sums of t-subnorms yielding t-norms were studied, and in [20, Theorem 3.1] it was shown that this construction is the most general way to obtain $\mathrm{t}$-norms as ordinal sums of semigroups.

THEOREM 2.5. Let $T$ be a t-norm. Then $([0,1], T)$ is an ordinal sum of semigroups if and only if $T$ is an ordinal sum of $t$-subnorms, that is, there exist a family $\left(*_{\alpha}\right)_{\alpha \in A}$ of $t$-subnorms and a family (]$a_{\alpha}, b_{\alpha}[)_{\alpha \in A}$ of pairwise disjoint open subintervals of $[0,1]$ such that, whenever $b_{\alpha_{0}}=1$ for some $\alpha_{0} \in A$, then $*_{\alpha_{0}}$ is a t-norm, and whenever $b_{\alpha_{0}}=a_{\beta_{0}}$ for some $\alpha_{0}, \beta_{0} \in A$ then either $*_{\alpha_{0}}$ is a t-norm or $*_{\beta_{0}}$ has no zero divisors, and such that

$$
T(x, y)= \begin{cases}a_{\alpha}+\left(b_{\alpha}-a_{\alpha}\right)\left(\frac{x-a_{\alpha}}{b_{\alpha}-a_{\alpha}} *_{\alpha} \frac{y-a_{\alpha}}{b_{\alpha}-a_{\alpha}}\right) & \text { if } \left.(x, y) \in] a_{\alpha}, b_{\alpha}\right]^{2} \\ \min (x, y) & \text { otherwise. }\end{cases}
$$

\section{Archimedean classes and components}

When investigating the structure of $t$-norms, their Archimedean subsemigroups play an important role (compare [9]).

Definition 3.1. Let $T$ be a t-norm. Two elements $x, y \in[0,1]$ are called Archimedean equivalent if there is an $n \in \mathbb{N}$ such that $x^{(n)} \leq y \leq x$ or $y^{(n)} \leq x \leq y$. For each $x \in[0,1]$, the equivalence class $I_{x}$ containing $x$ is called a $T$-Archimedean class of $T$ or Archimedean class if $T$ is either irrelevant or clear from context. 
Clearly, as noted in [14], each Archimedean class is a convex subset of [0,1]. Obviously, by complete analogy we may define the Archimedean classes of tosabs and, in particular, of t-subnorms.

\section{PROPOSITION 3.2. Let $T$ be a $t$-norm.}

(i) For all $(x, y) \in[0,1]^{2}$, we have $I_{T(x, y)}=I_{\min (x, y)}$.

(ii) For each $x \in[0,1]$, the pair $\left(I_{x},\left.T\right|_{I_{x}^{2}}\right)$ is a subsemigroup of $([0,1], T)$, and it is called an Archimedean component of $T$.

Proof. Property (i) follows from the fact that $T(x, x) \leq T(x, y) \leq x$ whenever $x \leq y$, and (ii) is immediate from (i).

As a consequence, for two t-norms $T_{1}$ and $T_{2}$ with the same Archimedean components we have $x_{T_{1}}^{(n)}=x_{T_{2}}^{(n)}$ for each $x \in[0,1]$ and $n \in \mathbb{N}$.

A necessary and sufficient condition for a singleton $\{x\}$ to be a (trivial) Archimedean class for a t-norm $T$ is that $T(y, z)=x$ holds if and only if $\min (y, z)=x$. As a consequence, $\{1\}$ is an Archimedean class of each t-norm $T$.

It is easy to see that a triangular norm is Archimedean if and only if its only non-trivial Archimedean class is either $[0,1[$ or $] 0,1[$.

Each Archimedean component $\left(I_{x},\left.T\right|_{l_{x}^{2}}\right)$ of $T$ is a tosab. As a consequence, Archimedean components can be used as summands in the construction given in Proposition 2.4, thus leading to a t-norm. From [18, Proposition 1.6 and Theorem 2.12] the following characterization of Archimedean components follows immediately.

LEMMA 3.3. A totally ordered abelian semigroup $(I, *)$ is an Archimedean component of some $t$-norm $T$ if and only if either $I=\{1\}$ or $I$ is a convex subset of $[0,1[$ such that for all $x \in I$ we have $\lim _{n \rightarrow \infty} x_{*}^{(n)}=\inf I$.

Observe that in the case $I=[0,1[$ or $I=] 0,1[$ the pair $(I, *)$ is an Archimedean component of some t-norm $T$ if and only if for each $x \in] 0,1\left[\right.$ we have $\lim _{n \rightarrow \infty} x^{(n)}=0$, that is, if and only if $T$ is Archimedean.

Clearly, for each t-norm the set of all Archimedean components forms a partition of $[0,1]$. Moreover, if for each non-empty subset $A \subseteq[0,1]$ we put $I_{A}=\bigcup_{x \in A} I_{x}$, then $\left(I_{A},\left.T\right|_{I_{A}^{2}}\right)$ is a totally ordered abelian semigroup whose semigroup operation is bounded from above by the minimum. That means, if the set $A$ is convex then $\left(I_{A},\left.T\right|_{I_{A}^{2}}\right)$ is a tosab. For each $x \in[0,1],\left(I_{x},\left.T\right|_{l_{x}^{2}}\right)$ is the maximal Archimedean subsemigroup containing $x$.

For an arbitrary t-norm $T$, the ordinal sum of its Archimedean components as introduced in Proposition 2.4 has the same Archimedean components as $T$. 
PROPOSITION 3.4. Let $T$ be a $t$-norm and $\left\{\left(I_{x},\left.T\right|_{l_{x}^{2}}\right) \mid x \in[0,1]\right\}$ the set of Archimedean components. Then the ordinal sum of the Archimedean components is the strongest t-norm which has the same Archimedean components as $T$.

Proof. Observe first that $\bigcup_{x \in[0,1]} I_{x}=[0,1]$, that the ordinal sum $*$ of the Archimedean components is a t-norm because of Proposition 2.4 and that $*$ and $T$ coincide on $\bigcup_{x \in[0,1]} I_{x}^{2}$. Therefore, the ordinal sum $*$ has the same Archimedean components as $T$. Moreover, since $*$ coincides on $[0,1]^{2} \backslash \bigcup_{x \in[0,1]} l_{x}^{2}$ with $T_{\mathrm{M}}$, $*$ is the strongest $\mathrm{t}$-norm with this property.

EXAMPLE 2. For the nilpotent minimum $T^{\mathrm{nM}}$ [18] given by

$$
T^{\mathbf{n M}}(x, y)= \begin{cases}0 & \text { if } x+y \leq 1 \\ \min (x, y) & \text { otherwise }\end{cases}
$$

the only non-trivial Archimedean component of $T^{\mathrm{nM}}$ is $\left([0,1 / 2], *_{1}\right)$ with $x *_{1} y=0$ for all $(x, y) \in[0,1 / 2]^{2}$. The ordinal sum $*$ of the Archimedean components of $T^{\mathrm{nM}}$ is given by

$$
x * y= \begin{cases}0 & \text { if }(x, y) \in[0,1 / 2]^{2} \\ \min (x, y) & \text { otherwise }\end{cases}
$$

Clearly, * and $T^{\mathrm{nM}}$ have the same Archimedean components. Moreover, for each set $A$ with $[0,1 / 2]^{2} \subseteq A \subseteq\left[0,1\left[^{2} \backslash\right] 1 / 2,1\left[^{2}\right.\right.$ satisfying, for each $(x, y) \in A$, both $(y, x) \in A$ and $[0, x] \times[0, y] \subseteq A$, the function $T_{A}:[0,1]^{2} \rightarrow[0,1]$ defined by

$$
T_{A}(x, y)= \begin{cases}0 & \text { if }(x, y) \in A \\ \min (x, y) & \text { otherwise }\end{cases}
$$

is a t-norm which has the same Archimedean components as $T^{\mathbf{n M}}$.

REMARK. (i) Because of Theorem 2.2, each continuous t-norm $T$ is uniquely determined by its non-trivial Archimedean components.

(ii) As a consequence, each continuous t-norm $T$ is uniquely determined when all the sequences $\left(x^{(n)}\right)_{n \in \mathbb{N}}$ are known (this was shown for strict t-norms in [17]).

(iii) Since the only non-trivial Archimedean classes of an Archimedean t-norm are $[0,1[$ or $] 0,1[$, two continuous $t$-norms with the same Archimedean classes are isomorphic. 


\section{Extension of Archimedean components to t-norms}

As just observed, a continuous t-norm is uniquely determined by its Archimedean components. Then, however, it is important to determine the Archimedean tosabs related to continuous t-norms. The proof of the following is obvious.

LEMMA 4.1. A non-trivial Archimedean tosab $(I, *)$ is an Archimedean component of some continuous $t$-norm $T$ if and only if either $I=[a, b[$ or $I=] a, b[$ for some $a, b \in[0,1], *$ is continuous and satisfies $\lim _{y>b} x * y=x$.

Note that, whenever a non-trivial Archimedean tosab $(I, *)$ with $I=[a, b[$ or $I=] a, b[$ is an Archimedean component of some continuous t-norm, then $*$ satisfies the conditional cancellation law, that is, $x * y=x * z>a$ implies $y=z$.

In general, starting with an arbitrary family of pairwise disjoint Archimedean tosabs $\left(\left(I_{\alpha}, *_{\alpha}\right)\right)_{\alpha \in A}$, by adding a family of trivial Archimedean tosabs $\left(\left(I_{\beta}, *_{\beta}\right)\right)_{\beta \in B}$ with $B=[0,1] \backslash \bigcup_{\alpha \in A} I_{\alpha}$ and $I_{\beta}=\{\beta\}$, we obtain a family of pairwise disjoint Archimedean tosabs $\left(\left(I_{\gamma}, *_{\gamma}\right)\right)_{\gamma \in A \cup B}$ such that $\left\{I_{\gamma} \mid \gamma \in A \cup B\right\}$ is a partition of $[0,1]$. Applying to this family $\left(\left(I_{\gamma}, *_{\gamma}\right)\right)_{\gamma \in A \cup B}$ of tosabs the same construction as in Proposition 3.4 , that is, defining the binary operation $*$ on $[0,1]$ by

$$
x * y= \begin{cases}x *_{\alpha} y & \text { if }(x, y) \in I_{\alpha}^{2} \\ \min (x, y) & \text { otherwise, }\end{cases}
$$

it is clear that $*$ is always a t-subnorm, and that $*$ is a t-norm if and only if $1 \notin \bigcup_{\alpha \in A} I_{\alpha}$. Obviously, $*$ is the strongest $\mathrm{t}$-subnorm (respectively $\mathrm{t}$-norm) having all the tosabs $\left(I_{\alpha}, *_{\alpha}\right)$ we started with as Archimedean components. However, as we have seen in Example 2,* is not necessarily the unique t-(sub)norm having all these Archimedean components.

The following result, whose proof is again straightforward, will be helpful for determining the uniqueness of $t$-norms with given Archimedean components.

LEMMA 4.2. Let $T$ be a t-norm and $\left\{I_{x} \mid x \in[0,1]\right\}$ the set of Archimedean components. Then the following are equivalent:

(i) For each t-norm $\tilde{T}$ with $\tilde{T} \neq T$, there is an element $x \in[0,1]$ such that the Archimedean component $\left(\tilde{I}_{x},\left.\tilde{T}\right|_{\left(\tilde{I}_{x}\right)^{2}}\right)$ of $\tilde{T}$ and the Archimedean component $\left(I_{x},\left.T\right|_{l_{x}^{2}}\right)$ of $T$ are different.

(ii) For all $(x, y) \in[0,1]^{2}$ with $x \leq y$, there is a unique totally ordered abelian semigroup $\left(I_{\{x, y\}}, *\right)$, where the operation $*$ is bounded from above by the minimum, such that both $\left(I_{x},\left.T\right|_{I_{x}^{2}}\right)$ and $\left(I_{y},\left.T\right|_{l_{y}^{2}}\right)$ are subsemigroups of $\left(I_{\{x, y\}}, *\right)$. 
Recall that $I_{\{x, y\}}=I_{\{x\}} \cup I_{\{y\}}$ and observe that, for each t-norm $T$ and for each $x \in[0,1]$ whose Archimedean class is a singleton (that is, $I_{x}=\{x\}$ ), assertion (ii) in Lemma 4.2 holds because of the monotonicity and boundary conditions of triangular norms. As a consequence, it suffices to consider non-trivial Archimedean classes. We shall look at some important special cases.

LEMMA 4.3. Assume that $I_{u}$ equals $\left[a, b[\right.$ or $] a, b\left[\right.$ and let $\left(I_{u}, *_{u}\right)$ be an Archimedean component of some $t$-norm $T$ such that for each $x \in] a, b[$ there is a $y \in] a, b[$ with $x *_{u} y>a$ and such that the conditional cancellation law holds. Then, putting $I=I_{u} \cup I_{b}$, the semigroup $\left(I,\left.T\right|_{l^{2}}\right)$ is the ordinal sum of $\left(I_{u}, *_{u}\right)$ and $\left(I_{b},\left.T\right|_{I_{b}^{2}}\right)$.

Proof. It suffices to show that $T(x, b)=x$ for all $x \in I_{u}$. Clearly, $b$ is an idempotent element of $T$. Suppose that we have $T(x, b)=v<x$ for some $x \in I_{u}$. Then necessarily $x \in] a, b\left[\right.$ and $x *_{u} y=T(x, y)>a$ for some $\left.y \in\right] a, b[$. Thus $a<T(x, y) \leq T(x, b)=v$, and we have $T(v, z)=v *_{u} z>a$ for some $\left.z \in\right] a, b[$. Moreover,

$$
\begin{aligned}
v *_{u} T(b, z) & =T(v, T(b, z))=T(T(x, T(b, b)), z) \\
& =T(T(x, b), z)=T(v, z)=v *_{u} z
\end{aligned}
$$

and, by cancellation, $T(b, z)=z$. Then also

$$
x *_{u} z=x *_{u} T(b, z)=T(T(x, b), z)=T(v, z)=v *_{u} z,
$$

implying $x=v$, contradicting our assumption.

Summarizing these results, we obtain the following sufficient condition for the uniqueness of $t$-norms with given Archimedean components.

THEOREM 4.4. Let $T$ be a t-norm and suppose that each of its non-trivial Archimedean components satisfies the hypotheses of Lemma 4.3. Then there is no other t-norm $\tilde{T}$ having the same Archimedean components as $T$.

Theorem 4.4 allows the relationship between continuous t-norms and their Archimedean components to be strengthened (not supposing explicitly the continuity of $T$ ).

COROLlaRY 4.5. Let $T$ be a t-norm, suppose that each of its non-trivial components is continuous and satisfies the hypotheses of Lemma 4.3 and, additionally, $\lim _{z>b_{x}} T(y, z)=y$ if $x \in[0,1], y \in I_{x}$ and $b_{x}=\sup I_{x}$. Then $T$ is a continuous t-norm, and it is uniquely determined by its Archimedean components.

Observe that the non-trivial components of the t-norms considered in Example 2 and [31, page 60] do not satisfy the hypotheses of Lemma 4.3. 
EXAMPLE 3. Assume that $T$ is a t-norm whose Archimedean components are $\left(\left[0,1 / 2\left[, *_{1}\right)\right.\right.$ with $x *_{1} y=x \cdot y,\left(\left[1 / 2,1\left[, *_{2}\right)\right.\right.$ with $x *_{2} y=1 / 2$, and the trivial component $(\{1\}, *)$. Then we get

$$
T(x, y)= \begin{cases}x \cdot y & \text { if }(x, y) \in\left[0,1 / 2\left[^{2}\right.\right. \\ 1 / 2 & \text { if }(x, y) \in\left[1 / 2,1\left[^{2}\right.\right. \\ \min (x, y) & \text { otherwise, }\end{cases}
$$

that is, $T$ necessarily is the ordinal sum of its Archimedean components.

As already observed, each family $\left(\left(I_{\alpha}, *_{\alpha}\right)\right)_{\alpha \in A}$ of pairwise disjoint Archimedean tosabs satisfying $I_{\alpha_{0}}=\{1\}$ for some $\alpha_{0} \in A$ whenever $1 \in \bigcup_{\alpha \in A} I_{\alpha}$ can be extended to a t-norm with no additional non-trivial Archimedean components by means of the ordinal sum construction (after filling, if necessary, the gaps in the given system of Archimedean tosabs by singleton components), and the resulting t-norm $T$ is the strongest t-norm coinciding, for each $\alpha \in A$, with $*_{\alpha}$ on $I_{\alpha}^{2}$. This fact is due to the simple observation that, for each subinterval $I$ of $[0,1],(I, *)$ with $x * y=\min (x, y)$ is the strongest tosab acting on $T$ and that it is an Archimedean component only if $I$ is a singleton. Clearly a weakest tosab acting on a subinterval $I$ of $[0,1]$ exists if $c=\inf I \in I$, in which case the semigroup operation $*: I^{2} \rightarrow I$ is given by $x * y=c$.

COROLLARY 4.6. Let $\left(\left(I_{\alpha}, *_{\alpha}\right)\right)_{\alpha \in A}$ be a family of pairwise disjoint Archimedean tosabs satisfying $I_{\alpha_{0}}=\{1\}$ for some $\alpha_{0} \in A$ whenever $1 \in \bigcup_{\alpha \in A} I_{\alpha}$, and assume that $[0,1\rceil \backslash \bigcup_{\alpha \in A} I_{\alpha}=\bigcup_{\beta \in B} J_{\beta}$ with $c_{\beta}=\inf J_{\beta} \in J_{\beta}$ and that there are no $\gamma, \delta \in B$ such that $\gamma \neq \delta$ and $J_{\gamma} \cup J_{\delta}$ is an interval. Then the function $T_{w}:[0,1]^{2} \rightarrow[0,1]$ given by

$$
T_{w}(x, y)= \begin{cases}x *_{\alpha} y & \text { if }(x, y) \in I_{\alpha}^{2} \\ c_{\beta} & \text { if }(x, y) \in J_{\beta}^{2} \\ \min (x, y) & \text { otherwise }\end{cases}
$$

is a t-norm. It is the weakest $t$-norm which coincides, for each $\alpha \in A$, with $*_{\alpha}$ on $I_{\alpha}^{2}$ and which can be written as an ordinal sum of tosabs.

If the hypotheses of Corollary 4.6 are satisfied then the t-norm $T_{w}$ given by (3) will be called a $w$-ordinal sum.

Let $\left(T_{\alpha}\right)_{\alpha \in A}$ be a family of t-norms and (]$a_{\alpha}, b_{\alpha}[)_{\alpha \in A}$ be a family of non-empty, pairwise disjoint open subintervals of $[0,1]$. The most important feature of the ordinal sum $T=\left(\left\langle a_{\alpha}, b_{\alpha}, T_{\alpha}\right\rangle\right)_{\alpha \in A}$ of t-norms [18] given by (2) is that $T$ coincides with the appropriate linear transformations of the t-norms $T_{\alpha}$ on the set $\bigcup_{\alpha \in A}\left[a_{\alpha}, b_{\alpha}\right]^{2}$. By filling gaps with the minimum, we obviously obtain the strongest possible t-norm 
which coincides with such linear transformations. If the topological closure of the set $\bigcup_{\alpha \in A}\left[a_{\alpha}, b_{\alpha}\right]$ is a proper subset of $[0,1]$, it is always possible to construct other t-norms which are ordinal sums of semigroups which coincide with linear transformations on $\bigcup_{\alpha \in A}\left[a_{\alpha}, b_{\alpha}\right]^{2}$.

Recall that the set $\left[0,1\left[\backslash \bigcup_{\alpha \in A}\left[a_{\alpha}, b_{\alpha}[\right.\right.\right.$ can always be written as the union of pairwise disjoint intervals $\bigcup_{\beta \in B} Y_{\beta}$, where each $Y_{\beta}$ is a component with respect to connectedness containing a smallest element, that is, each $Y_{\beta}$ is of the form $\left[c_{\beta}, d_{\beta}\right.$ [ or $\left[c_{\beta}, d_{\beta}\right]$.

COROLlaRY 4.7. Let $T=\left(\left\langle a_{\alpha}, b_{\alpha}, T_{\alpha}\right\rangle\right)_{\alpha \in A}$ be an ordinal sum of $t$-norms. Then the $w$-ordinal sum $T_{w}:[0,1]^{2} \rightarrow[0,1]$ of the summands $\left\langle a_{\alpha}, b_{\alpha}, T_{\alpha}\right\rangle$ given by

$$
T_{w}(x, y)= \begin{cases}a_{\alpha}+\left(b_{\alpha}-a_{\alpha}\right) T_{\alpha}\left(\frac{x-a_{\alpha}}{b_{\alpha}-a_{\alpha}}, \frac{y-a_{\alpha}}{b_{\alpha}-a_{\alpha}}\right) & \text { if }(x, y) \in\left[a_{\alpha}, b_{\alpha} L^{2} ;\right. \\ c_{\beta} & \text { if }(x, y) \in Y_{\beta}^{2} ; \\ \min (x, y) & \text { otherwise, }\end{cases}
$$

is the weakest t-norm which is an ordinal sum of semigroups and which contains all the original summands of $T$.

PROOF. This follows directly from Proposition 2.4 taking into account that the weakest semigroup operation $*_{\beta}$ on $Y_{\beta}$ is given by $x *_{\beta} y=c_{\beta}$.

Obviously, all non-trivial Archimedean components of $T$ are also $T_{w}$-Archimedean. However, additional non-trivial $T_{w}$-Archimedean components occur if at least one interval $Y_{\beta}$ is non-trivial. Clearly $T_{w}=T$ in Corollary 4.7 if and only if $[0,1]$ equals the topological closure of $\bigcup_{\alpha \in A}\left[a_{\alpha}, b_{\alpha}\right]$.

In general, the $w$-ordinal sum $T_{w}$ cannot be written as an ordinal sum of t-norms containing all the original summands: for example, starting with the ordinal sum of t-norms

$$
T=\left(\left\langle\frac{n+4}{2 n+4}, \frac{n+3}{2 n+2}, T_{\mathrm{M}}\right\rangle\right)_{n \in \mathbb{N}}
$$

then $T_{w}$ coincides with the t-norm $*$ given in Example 2, which is not an ordinal sum of t-norms (only an ordinal sum of t-subnorms) containing all the original summands. However, for a finite index set $A=\{1,2, \ldots, n\}$ the $w$-ordinal sum $T_{w}$ turns out to be an ordinal sum of t-norms containing the original summands. Put $B=\{0,1\} \cup$ $\left\{a_{1}, b_{1}, a_{2}, b_{2}, \ldots, a_{n}, b_{n}\right\}=\left\{c_{0}, c_{1}, \ldots, c_{m}\right\}$ with $0=c_{0}<c_{1}<\cdots<c_{m}=1$ and, for $i \in\{1,2, \ldots, m\}$,

$$
\tilde{T}_{i}= \begin{cases}T_{j} & \text { if } a_{j}=c_{i-1} \\ T_{\mathbf{D}} & \text { otherwise }\end{cases}
$$


where $T_{\mathbf{D}}$ is the drastic product. Then $T_{w}$ can be written as an ordinal sum of t-norms as follows:

$$
T_{w}=\left(\left\langle c_{i-1}, c_{i}, \tilde{T}_{i}\right\rangle\right)_{i \in[1,2, \ldots, m\}} .
$$

Note that in (4) the t-norm $T_{\mathrm{D}}$ can be replaced by an arbitrary t-norm and formula (5) still will yield a t-norm which coincides with $T$ on the square $\left[a_{i}, b_{i}\right]^{2}$ for each $i \in\{1,2, \ldots, n\}$. If, for example, we replace $T_{\mathrm{D}}$ by the Eukasiewicz $\mathrm{t}$-norm $T_{\mathrm{L}}$ and if $T_{1}, T_{2}, \ldots, T_{n}$ are all (associative) copulas [19,27], then formula (5) gives the weakest (associative) copula containing all the summands $\left\langle a_{i}, b_{i}, T_{i}\right\rangle$.

Observe that the $w$-ordinal sum $T_{w}$ given in Corollary 4.7 is the weakest $\mathrm{t}$-norm coinciding with $T$ on the square $\left[a_{\alpha}, b_{\alpha}\right]^{2}$ for each $\alpha \in A$ if and only if the topological closure of $\bigcup_{\alpha \in A}\left[a_{\alpha}, b_{\alpha}\right]$ is a closed interval of the form $[0, c]$ for some $\left.\left.c \in\right] 0,1\right]$. It was shown in [6] that the weakest $\mathrm{t}$-norm $\tilde{T}$ coinciding with $T$ on the square $\left[a_{\alpha}, b_{\alpha}\right]^{2}$ for each $\alpha \in A$ (which in general is not an ordinal sum of semigroups containing all the original summands) is given by

$$
\tilde{T}(x, y)=\left\{\begin{array}{cc}
\sup \left\{a_{\alpha}+\left(b_{\alpha}-a_{\alpha}\right) T_{\alpha}\left(\frac{\min \left(b_{\alpha}, x\right)-a_{\alpha}}{b_{\alpha}-a_{\alpha}}, \frac{\min \left(b_{\alpha}, y\right)-a_{\alpha}}{b_{\alpha}-a_{\alpha}}\right)\right. \\
\left.\alpha \in A, a_{\alpha} \leq \min (x, y)\right\} \quad \text { if } \max (x, y)<1 \\
\min (x, y) & \text { otherwise. }
\end{array}\right.
$$

Note that, using the notation of Corollary 4.7 , the t-norm $\tilde{T}$ can be rewritten as follows:

$$
\tilde{T}(x, y)=\left\{\begin{array}{lc}
a_{\alpha}+\left(b_{\alpha}-a_{\alpha}\right) T_{\alpha}\left(\frac{x-a_{\alpha}}{b_{\alpha}-a_{\alpha}}, \frac{y-a_{\alpha}}{b_{\alpha}-a_{\alpha}}\right) & \text { if }(x, y) \in\left[a_{\alpha}, b_{\alpha} L^{2} ;\right. \\
c_{\beta} & \text { if } \min (x, y) \in Y_{\beta} \text { and } \\
\min (x, y) & \max (x, y)<1 ; \\
\text { otherwise. }
\end{array}\right.
$$

\section{Construction of Archimedean components}

As we have seen in Section 4, Archimedean components (tosabs) are, on one hand, essential tools when constructing triangular norms. Indeed, the knowledge of all Archimedean components of a t-norm induces full information about the trajectories $\left(x^{(n)}\right)_{n \in \mathbb{N}}$ for each $\left.x \in\right] 0,1[$. To pass from the trajectories to the Archimedean components is possible in special cases only, for example, if the $t$-norm is continuous. It is for this reason that the trajectories of a continuous t-norm determine this $t$-norm uniquely [17] (compare Remark 3). 
On the other hand, in order to construct new t-norms (for example, as ordinal sums of semigroups) it is important to know a rich variety of possible Archimedean components.

LEMMA 5.1. Let $(G, \preceq, *)$ be an abelian, totally ordered Archimedean semigroup. Then for all $u, v \in G$ we have $u * v \preceq u$, that is, $*$ is bounded above by the minimum operator induced by $\preceq$.

PROOF. Suppose to the contrary that there exist $u, v \in G$ such that $u \prec u * v$. By a simple induction $u \prec u * v^{(n)}$ for all $n \in \mathbb{N}$. Since $*$ is Archimedean, $v^{(p)} \preceq u$ and $u^{(2 q)}=(u * u)^{(q)} \preceq u$ for some $p, q \in \mathbb{N}$. In particular $u \prec u * v^{(p)} \preceq u * u$. Again, by a simple induction, $u \prec u^{(n)}$ for all $n \geq 2$, so, in particular, $u \prec u^{(2 p)} \preceq u$, a contradiction.

Because of Lemma 5.1 it is clear that, given an abelian, totally ordered Archimedean semigroup $(G, \preceq, *)$, a subinterval $I$ of $[0,1[$ and an order preserving bijection $\varphi$ : $I \rightarrow G$, then also $\left(I, \leq, *_{\varphi}\right)$, where $*_{\varphi}$ is given by $x *_{\varphi} y=\varphi^{-1}(\varphi(x) * \varphi(y))$, is a tosab and hence an Archimedean component of some t-norm. A semigroup $(G, \preceq, *)$ which satisfies the hypotheses of Lemma 5.1 and which is isomorphic to some tosab will be called a fitting Archimedean semigroup.

EXAMPLE 4. (i) The semigroup (] $1, \infty[, \preceq,+)$ with $x \preceq y$ if and only if $x \geq y$ is a fitting Archimedean semigroup: the pair (]$a, b\left[,+_{\varphi}\right)$ is an Archimedean component for each open subinterval $] a, b[$ of $[0,1[$ and for each order preserving bijection $\varphi:(] a, b[, \leq) \rightarrow(] 1, \infty[, \preceq)$.

(ii) Coming back to the t-norms $T_{1}$ and $T_{2}$ in Example 1, equip the set $G$ of strictly increasing sequences of positive integers which are proper subsequences of $(n)_{n \in \mathbb{N}}$ with the inverse lexicographic order $\preceq$, that is, $\left(x_{n}\right)_{n \in \mathbb{N}} \preceq\left(y_{n}\right)_{n \in \mathbb{N}}$ if and only if there is an $n_{0} \in \mathbb{N}$ such that $x_{n_{0}}>y_{n_{0}}$ and $x_{n}=y_{n}$ for all $n<n_{0}$. Defining the operation $*$ on $G$ by $\left(x_{n}\right)_{n \in \mathbb{N}} *\left(y_{n}\right)_{n \in \mathbb{N}}=\left(x_{n}+y_{n}\right)_{n \in \mathbb{N}}$ and the order preserving bijection $\varphi:(] 0,1[, \leq) \rightarrow(G, \preceq)$ by $\varphi^{-1}\left(\left(x_{n}\right)_{n \in \mathbb{N}}\right)=\sum_{n=1}^{\infty} 1 / 2^{x_{n}}$, then $(G, *)$ is a fitting Archimedean semigroup and (] $0,1\left[,\left.T_{1}\right|_{0,1\left[^{2}\right.}\right)$ is a $T_{1}$-Archimedean component which is isomorphic to $(G, *)$.

Now, put $H=G \cup\left\{(n)_{n \in \mathbb{N}}\right\}$, introduce the order $\sqsubseteq$ on $H$ by keeping the original order $\preceq$ on $G$ and by supposing that the sequence $(n)_{n \in \mathbb{N}}$ be the greatest element of $H$, and define the binary operation o on $H$ by $\left(x_{n}\right)_{n \in \mathbb{N}} \circ\left(y_{n}\right)_{n \in \mathbb{N}}=\left(x_{n}+y_{n}-n+1\right)_{n \in \mathbb{N}}$. Then, for a fixed $\left.\left.m \in \mathbb{N}, \psi:(] 1-1 / 2^{m-1}, 1-1 / 2^{m}\right], \leq\right) \rightarrow(H$, 드) given by

$$
\psi^{-1}\left(\left(x_{n}\right)_{n \in \mathbb{N}}\right)=1-\frac{1}{2^{m-1}}+\sum_{n=1}^{\infty} \frac{1}{2^{m+x_{n}}}
$$


is an order preserving bijection, and (] $\left.\left.1-1 / 2^{m-1}, 1-1 / 2^{m}\right], o_{\psi}\right)$ is a $T_{2}$-Archimedean component.

We present here three further ways for producing Archimedean components. The first has a straightforward proof, the second uses pseudo-inverses, and the third starts from a finite semigroup.

PROPOSITION 5.2. Let $(I, *)$ be an Archimedean component, let $x \in I$ and put $J_{x}=[0, x] \cap I$ and $K_{x}=\left[0, x\left[\cap I\right.\right.$. Then $\left(J_{x},\left.*\right|_{J_{x}^{2}}\right)$ and $\left(K_{x},\left.*\right|_{K_{x}^{2}}\right)$ are Archimedean components.

PROPOSITION 5.3. Let I be a subinterval of $[0,1[$ and let $f: I \rightarrow] 0, \infty[$ be a continuous non-increasing function which is unbounded if inf $I \notin I$. Define the operation $*$ on I by

$$
x * y=f^{(-1)}(f(x)+f(y)),
$$

where $f^{(-1)}$ is the pseudo-inverse of $f$. Then $(I, *)$ is an Archimedean component, and the semigroup operation $*$ is left-continuous.

PROOF. Observe first that $*$ is well defined. Also, $f^{(-1)}$ is right-continuous, and the continuity of $f$ implies the strict monotonicity of $f^{(-1)}$ on the range of $f$ and also $f \circ f^{(-1)}(x)=x$ for all $x$ in the range of $f$. Since the range of $f$ is an interval, the operation $*$ is associative and we have $x_{*}^{(n)}=f^{(-1)}(n \cdot f(x))$, implying $\lim _{n \rightarrow \infty} x_{*}^{(n)}=\inf I$ for each $x \in I$. The rest of the proof is straightforward.

In combination with Theorem 2.5, the method described in Proposition 5.3 allows large classes of left-continuous t-norms to be constructed as follows (recall that exactly the left-continuous t-norms possess a residuum which is often used as interpretation of the implication in many-valued and fuzzy logics [11, 13]): given an arbitrary family (]$a_{\alpha}, b_{\alpha}[)_{\alpha \in A}$ of pairwise disjoint open subintervals of $[0,1]$ and a family $\left(f_{\alpha}\right.$ : $\left.\left[a_{\alpha}, b_{\alpha}\right] \rightarrow[0, \infty]\right)_{\alpha \in A}$ of continuous non-increasing functions satisfying $f_{\alpha}(1)=0$ whenever $b_{\alpha}=1$ and such that, if $b_{\alpha}=a_{\beta}$ for some $\alpha, \beta \in A, f_{\beta}\left(a_{\beta}\right)$ is finite only if $f_{\alpha}\left(b_{\alpha}\right)=0$, then the function $T:[0,1]^{2} \rightarrow[0,1]$ given by

$$
T(x, y)= \begin{cases}f_{\alpha}^{(-1)}\left(f_{\alpha}(x)+f_{\alpha}(y)\right) & \text { if } \left.(x, y) \in] a_{\alpha}, b_{\alpha}\right]^{2} \\ \min (x, y) & \text { otherwise, }\end{cases}
$$

is a left-continuous t-norm.

PROPOSITION 5.4. Consider a finite set $G$ with cardinality $n$ and an abelian, totally ordered Archimedean semigroup $(G, 0)$ (observe that $\circ$ is necessarily nilpotent). For 
each subinterval $\left[a, b\left[\right.\right.$ of $[0,1]$ and for all n-element subsets $A=\left\{a_{0}, a_{1}, \ldots, a_{n-1}\right\}$ with $a=a_{0}<a_{1}<\cdots<a_{n-1}<b$, let $(A, \diamond)$ be the abelian, totally ordered Archimedean semigroup which is isomorphic to $(G, 0)$. Putting $x * y=x_{A} \diamond y_{A}$, where $x_{A}=\max \{u \in A \mid u \leq x\}$, then $([a, b[, *)$ is an Archimedean component, and the semigroup operation $*$ is right-continuous.

Proof. The proof follows from $(x * y)_{A}=x_{A} \diamond y_{A}$ and from the right continuity of the function $\tau:\left[a, b\left[\rightarrow A\right.\right.$ defined by $\tau(x)=x_{A}$.

REMARK. (i) If an Archimedean component $(I, *)$ has an additive generator $f: l \rightarrow] 0, \infty\left[\right.$ and if the Archimedean components $\left(J_{x},\left.*\right|_{J_{x}^{2}}\right)$ and $\left(K_{x},\left.*\right|_{K_{x}^{2}}\right)$ are obtained as in Proposition 5.2, then these semigroups are generated by the additive generators $\left.f\right|_{J_{x}}$ and $\left.f\right|_{K_{x}}$, respectively.

(ii) As a consequence of [25] we have: if in an Archimedean component $(I, *)$ the operation $*$ has an additive generator $f: I \rightarrow] 0, \infty[$, then $*$ is continuous if and only if the function $f$ is strictly monotone on the set $\{x \in I \mid$ there is some $y \in I$ such that $x \leq y * y\}$.

EXAmPLE 5. Consider the function $f:[0,1[\rightarrow[0, \infty]$ which is given by $f(x)=$ $\max (10-18 x, 1)$. Because of (ii) above the operation $*$ on $[0,1[$ given by (6) is continuous. According to Proposition 5.3, $([0,1[, *)$ is an Archimedean component of the uniquely determined t-norm $T$ given by

$$
T(x, y)= \begin{cases}\min (x, y) & \text { if } \max (x, y)=1 \\ \max \left(\min \left(x, \frac{1}{2}\right)+\min \left(y, \frac{1}{2}\right)-\frac{5}{9}, 0\right) & \text { otherwise. }\end{cases}
$$

Putting $x_{0}=1 / 2$ we obtain $J_{x_{0}}=[0,1 / 2]$ and $\left.f\right|_{J_{0}}(x)=10-18 x$. Obviously, $\left.f\right|_{x_{x_{0}}}$ generates $\left.*\right|_{x_{00}^{2}}$ (which coincides with $\left.T\right|_{\delta_{x_{0}}^{2}}$ ).

\section{Concluding remarks}

Continuous t-norms are just ordinal sums of continuous Archimedean t-norms, the latter being generated by continuous additive generators (see Theorems 2.2 and 2.1). Left-continuous t-norms and the corresponding residual implications (which are linked by the Galois connection [10]) play a crucial role in many-valued logics, but no characterization of left-continuous t-norms is known so far. Concerning the relationship between left-continuous and continuous $t$-norms, note that left-continuous $t$-norms which are either Archimedean or which are generated by some additive generator are necessarily continuous $[18,22,33]$. Proposition 5.3, applied to continuous Archimedean components (that is, to special threads [5, 15], for example to Archimedean 
components which are generated by continuous additive generators), gives rise to new left-continuous t-norms. Such left-continuous t-norms can be characterized in very special cases only, for example, if their Archimedean components satisfy the cancellation law in which case they are generated because of [1]. However, if the Archimedean components of such left-continuous t-norms cannot be extended to $I$ semigroups $[7,26,28]$ or if they do not satisfy the cancellation law, the characterization of those $\mathrm{t}$-norms is still an open problem.

\section{References}

[1] J. Aczél, Lectures on functional equations and their applications (Academic Press, New York, 1966).

[2] D. Butnariu and E. P. Klement, Triangular norm-based measures and games with fuzzy coalitions (Kluwer Academic Publishers, Dordrecht, 1993).

[3] J. H. Carruth, J. A. Hildebrant and R. J. Koch, The theory of topological semigroups, Monographs Textbooks Pure Appl. Math. 75 (Marcel Dekker, New York, 1983).

[4] A. H. Clifford, 'Naturally totally ordered commutative semigroups', Amer. J. Math. 76 (1954), 631-646.

[5] — 'Connected ordered topological semigroups with idempotent endpoints. I.', Trans. Amer. Math. Soc. 88 (1958), 80-98.

[6] B. De Baets and R. Mesiar, 'Ordinal sums of aggregation operators', in: Technologies for constructing intelligent systems. 2: Tools (eds. B. Bouchon-Meunier, J. Gutiérrez-Ríos, L. Magdalena and R. R. Yager) (Physica, Heidelberg, 2002) pp. 137-148.

[7] W. M. Faucett, 'Compact semigroups irreducibly connected between two idempotents', Proc. Amer. Math. Soc. 6 (1955), 741-747.

[8] J. C. Fodor and M. Roubens, Fuzzy preference modelling and multicriteria decision support (Kluwer Academic Publishers, Dordrecht, 1994).

[9] L. Fuchs, Partially ordered algebraic systems (Pergamon Press, Oxford, 1963).

[10] G. Gierz, K. H. Hofmann, K. Keimel, J. D. Lawson, M. W. Mislove and D. S. Scott, A Compendium of continuous lattices (Springer, Berlin, 1980).

[11] S. Gottwald, A treatise on many-valued logic, Stud. Logic Comput. (Research Studies Press, Baldock, 2001).

[12] O. Hadžić and E. Pap, Fixed point theory in probabilistic metric spaces (Kluwer Academic Publishers, Dordrecht, 2001).

[13] P. Hájek, Metamathematics of fuzzy logic (Kluwer Academic Publishers, Dordrecht, 1998).

[14] I. W. Hion, 'Ordered semigroups', Izv. Akad. Nauk SSSR 21 (1957), 209-222 (Russian).

[15] K. H. Hofmann and J. D. Lawson, 'Linearly ordered semigroups: Historic origins and A. H. Clifford's influence', in: Semigroup theory and its applications (eds. K. H. Hofmann and M. W. Mislove), London Math. Soc. Lecture Notes 231 (Cambridge University Press, Cambridge, 1996) pp. 15-39.

[16] S. Jenei, 'Structure of left-continuous triangular norms with strong induced negations. (I) Rotation construction', J. Appl. Non-Classical Logics 10 (2000), 83-92.

[17] C. Kimberling, 'On a class of associative functions', Publ. Math. Debrecen 20 (1973), 21-39.

[18] E. P. Klement, R. Mesiar and E. Pap, Triangular Norms (Kluwer Academic Publishers, Dordrecht, 2000). 
[19] _ 'Uniform approximation of associative copulas by strict and non-strict copulas', Illinois J. Math. 45 (2001), 1393-1400.

[20] _- 'Triangular norms as ordinal sums of semigroups in the sense of A. H. Clifford', Semigroup Forum 65 (2002), 71-82.

[21] E. P. Klement and S. Weber, 'Fundamentals of a generalized measure theory', in: Mathematics of Fuzzy Sets. Logic, Topology, and Measure Theory (eds. U. Höhle and S. E. Rodabaugh), The Handbook of Fuzzy Sets Series (Kluwer Academic Publishers, Boston, 1999) pp. 633-651.

[22] A. Kolesárová, 'A note on Archimedean triangular norms', BUSEFAL 80 (1999), 57-60.

[23] C. M. Ling, 'Representation of associative functions', Publ. Math. Debrecen 12 (1965), 189-212.

[24] K. Menger, 'Statistical metrics', Proc. Nat. Acad. Sci. U.S.A. 8 (1942), 535-537.

[25] A. Mesiarová, 'Continuous triangular subnorms', Fuzzy Sets and Systems, 142 (2004), 75-83.

[26] P. S. Mostert and A. L. Shields, 'On the structure of semi-groups on a compact manifold with boundary', Ann. of Math., II. Ser. 65 (1957), 117-143.

[27] R. B. Nelsen, An introduction to copulas, Lecture Notes in Statistics 139 (Springer, New York, 1999).

[28] A. B. Paalman-de Miranda, Topological semigroups, Mathematical Centre Tracts 11 (Matematisch Centrum, Amsterdam, 1964).

[29] E. Pap, Null-additive set functions (Kluwer Academic Publishers, Dordrecht, 1995).

[30] B. Schweizer and A. Sklar, 'Statistical metric spaces', Pacific J. Math. 10 (1960), 313-334.

[31] __ Probabilistic metric spaces (North-Holland, New York, 1983).

[32] B. Schweizer and J. Smítal, 'Measures of chaos and a spectral decomposition of dynamical systems on the interval', Trans. Amer. Math. Soc. 344 (1994), 737-754.

[33] P. Viceník, 'Additive generators of non-continuous triangular norms', in: Topological and Algebraic Structures in Fuzzy Sets: A Handbook of Recent Developments in the Mathematics of Fuzzy Sets (eds. S. E. Rodabaugh and E. P. Klement) (Kluwer Academic Publishers, Dordrecht, 2003) pp. $441-454$.

[34] L. A. Zadeh, 'Fuzzy sets', Inform. and Control 8 (1965), 338-353.

Department of Algebra

Stochastics and Knowledge-Based

Mathematical Systems

Johannes Kepler University

A-4040 Linz

Austria

e-mail: ep.klement@jku.at

Department of Mathematics

and Informatics

University of Novi Sad,

YU-21000 Novi Sad,

Serbia and Montenegro

e-mail: pap@im.ns.ac.yu

pape@eunet.yu
Department of Mathematics and Descriptive Geometry

Faculty of Civil Engineering Slovak University of Technology SK-81 368 Bratislava Slovakia and

Institute of Information Theory and Automation Czech Academy of Sciences CZ-18208 Prague 8 Czech Republic e-mail: mesiar@math.sk 